\title{
Regression-based prediction of seeking diabetes-related emergency medical assistance by regular clinic patients
}

\author{
Wasantha P. Jayawardene ${ }^{1}$ - Dayani C. Nilwala ${ }^{2} \cdot$ Godfred O. Antwi $^{1} \cdot$ David K. Lohrmann $^{1}$ • \\ Mohammad R. Torabi ${ }^{1} \cdot$ Stephanie L. Dickinson $^{3}$
}

Received: 11 January 2017 / Accepted: 14 August 2017 / Published online: 14 September 2017

(C) Research Society for Study of Diabetes in India 2017

\begin{abstract}
This study aims to examine diabetes-related emergency medical assistance (DREMA)-seeking factors among type-2 diabetes patients and predict likelihood of patients seeking DREMA before their next scheduled clinic visit. This case-control-designed study comprised a systematic random sampling of 228 patients who completed a structured interview (mean age $=62.6$ years). DREMA prediction model was developed based on parameter estimates of a logistic regression model on DREMA ( $\geq 1$ admission vs. 0 admissions), with variable selection from a forward stepwise selection process, considering all 24 potential independent variables. For the final DREMA prediction model, four variables were retained via forward stepwise logistic regression analysis: (1) age, (2) type of rice consumed, (3) physical activity outside of a regular job, and (4) leisure time exercise frequency. Likelihood of seeking DREMA increased with aging, regular or frequent consumption of white rice rather than brown or parboiled rice, and being physically inactive outside of occupation. Odds of seeking DREMA were directly associated with frequency of exercise during leisure time. With further validation and model updating based on local population characteristics, clinicians will be able to predict the DREMA-event likelihood for each clinic patient diagnosed with type-2
\end{abstract}

Godfred O. Antwi

gantwi@indiana.edu

1 Applied Health Science, School of Public Health Bloomington, Indiana University, Bloomington, IN, USA

2 Pathology Department, Base Hospital Homagama, Homagama, Sri Lanka

3 Epidemiology and Biostatistics, School of Public Health Bloomington, Indiana University, Bloomington, IN, USA diabetes. Modifiable DREMA-seeking variables suggest possible interventions for preventing undesired DREMA events.

Keywords Type-2 diabetes $\cdot$ Clinic patients $\cdot$ Logistic regression $\cdot$ Prediction $\cdot$ Diabetes-related emergency medical assistance

\section{Introduction}

Diabetes is a costly worldwide pandemic. Many patients diagnosed with type-2 diabetes mellitus have associated complications, including cardiovascular diseases, nephropathy, neuropathy, and foot ulcers, all of which can generate emergency conditions [1-4]. For diabetes alone, occurrence of hyperglycemia and hypoglycemia primarily leads to medical emergencies [5-8]. As paramedic emergency services are limited to trauma care [9], only three types of diabetes-related emergency medical assistance (DREMA) are available for diabetes patients in many low-income countries like Sri Lanka, visit the following: a family practitioner; the outpatient department of a public or private hospital; or the OPD of a hospital with subsequent transfer to emergency room, inpatient department (non-elective hospitalization), or a larger hospital. All three options will be hereafter referred to as "DREMA," without specifying the form of assistance.

Both meaningful and accurate validated mathematical clinical prediction models have added advantages over human clinical prediction approaches [10]. Mathematical models can integrate more factors than a clinician is capable of considering during decision making [11]. Although human clinical decision making is inconsistent and biased, especially when time and/or clinician's experience are limited, mathematical models consistently produce the same output with identical data inputs $[11,12]$. Furthermore, empirical research 
findings demonstrate that several prediction models are more accurate than human clinical prediction [13]. Models based on multivariate logistic regression provide output in the form of odds ratios (odds of an event occurring vs. the odds that it will not), are user-friendly and interpretable with a less arbitrary nature of assigning weights compared with univariate models $[14,15]$.

This study aimed to retrospectively examine factors that contributed to worsening of symptoms among type-2 diabetes patients who attended a suburban medical clinic in Sri Lanka and, utilizing a user-friendly logistic regression model, to predict the likelihood of a patient seeking DREMA before his/her next visit to a clinic within 3 months. Patients with either cardiovascular complications (e.g., hypertension, a history of myocardial infarction) or other comorbidities (e.g., asthma, epilepsy) and those with type- 1 diabetes were excluded in order to reduce the number of testable hypotheses as well as to simplify the final prediction model [16].

\section{Methods}

Conceptual model Based on empirical research evidence, an informal model was developed using a set of logical hypotheses. Likelihood of preventing DREMA was viewed as a complex outcome of general health status, pre-existing condition of diabetes, pre-disposing factors (e.g., age, education), reinforcing factors (e.g., social norms), and enabling factors (e.g., diabetes education) [17-19]. Variables that were thought to influence DREMA seeking were categorized under six domains: (1) anthropometry and blood sugar (measured at many diabetes clinics), (2) demography and occupational posture, (3) diabetes history and management, (4) food types and intake frequency, (5) physical activity and exercise, and (6) smoking and alcohol consumption.

Study setting The study was conducted in the medical clinic of Homagama Base Hospital, Sri Lanka located in a suburb of the capital, Colombo. The medical clinic had been held twice every week, whereas each clinic patient was expected to have follow-up visits at least once every 3 months for consultation and treatment - $\mathrm{a}$ free service. The Institutional Review Board of Indiana University, USA, approved the recruitment of human subjects in this study.

Study design Case-control study design was utilized in a medical clinic [20]. The study compared type-2 diabetes patients who had DREMA between two subsequent clinic visits with type-2 diabetes patients who had no DREMA during a 6month period between February and July. The study design involved the retrospective collection of behavioral data (corresponding to the time period prior to previous clinic visit) and retrospective review of clinic records for fasting blood sugar level (during previous clinic visit), along with on-site measurement of weight, height, waist, and hips.

Study population Approximately a one third of medical clinic patients had type-2 diabetes. The study population consisted of clinic patients who had diagnosed type-2 diabetes mellitus but had no other chronic illness (e.g., asthma, epilepsy). Patients were recruited when they came to the pathology laboratory for the fasting blood glucose (FBG) test prior to visiting the clinic.

Sampling method Based on these established exclusion criteria, slightly less than half of type-2 diabetes patients were eligible. As clinic procedures required all diabetes patients to complete a FBG prior to follow-up management during their visit, FBG testing was the mechanism used to systematically draw a random sample. Hence, every fifth diabetes patient who arrived for FBG testing was invited to participate in the study. The first interviewee of each clinic day was selected by using random numbers.

Instrument Data were collected through use of a 31-item structured interview administered by a qualified physician. A study information sheet was administered to participants prior to the interview and special consent was obtained to examine clinic records in addition to the informed consent. The survey was originally developed for this study and had no prior validation. Survey questions were formulated by four experts representing four different disciplines (medicine, health behavior, nutrition, and biostatistics) and were based on evidence from literature and work experience in similar clinics. Interview questions revealed information about the variables of interest in the conceptual model. All questions were appropriate to the culture and the health-related behaviors of urban Sri Lanka.

Data collection Data collection occurred during regular morning sessions of the twice weekly clinic. During the informed consent process, 228 of the 251 total invited clinic patients responded to the structured interview (Table 1), yielding a high response rate of 90.8\% during the 3-month survey period. The interviewer-a physician working in the pathology laboratory of the same hospital and not a diabetes clinic service provider - clarified the patients' answers and recorded them appropriately. In order to minimize patients' possible response biases, interviews were carried outside the diabetes clinic but within the hospital. Any contradicting or confusing information provided by a patient was resolved by consulting his/her clinic records in order to minimize interviewer bias and response bias. Total time taken for interview, anthropometry, and clinic records review was approximately $30 \mathrm{~min}$.

Data analysis Data were input into Excel and then analyzed with IBM SPSS statistical software, version 24.0; 18 of the 24 independent variables were treated as categorical data (see Table 1 for details). The dependent variable, DREMA was coded as 0 admissions vs. 1 or more admissions. Pearson Chi-square tests $\left(\chi^{2}\right)$ were 
Table 1 Description of Variables and Associations between DREMA and 24 Independent Variables (18 Categorical and 6 Continuous)

\begin{tabular}{|c|c|c|c|c|c|c|c|}
\hline \multirow{3}{*}{$\begin{array}{l}\text { Variables } \\
\text { Categorical variables }\end{array}$} & & & \multicolumn{5}{|c|}{ EMA (Emergency Medical Assistance) } \\
\hline & & & \multicolumn{2}{|c|}{0 Admission } & \multicolumn{3}{|c|}{$>=1$ Admission } \\
\hline & & Overall frequency & $\mathrm{n}$ & $\%$ & $\mathrm{n}$ & $\%$ & $p$ value \\
\hline \multirow[t]{2}{*}{ Gender } & Female & 113 & 93 & $82.3 \%$ & 20 & $17.7 \%$ & 0.281 \\
\hline & Male & 115 & 88 & $76.5 \%$ & 27 & $23.5 \%$ & \\
\hline \multirow[t]{3}{*}{ Posture at work } & Sitting and standing & 121 & 93 & $76.9 \%$ & 28 & $23.1 \%$ & $<.001$ \\
\hline & Walking & 97 & 79 & $81.4 \%$ & 18 & $18.6 \%$ & \\
\hline & Heavy physical work & 10 & 9 & $90.0 \%$ & 1 & $10.0 \%$ & \\
\hline \multirow[t]{2}{*}{ Having regular clinic visits? } & Yes & 168 & 134 & $79.8 \%$ & 34 & $20.2 \%$ & 0.814 \\
\hline & No & 60 & 47 & $78.3 \%$ & 13 & $21.7 \%$ & \\
\hline \multirow[t]{2}{*}{ Diabetes education } & Yes & 120 & 96 & $80.0 \%$ & 24 & $20.0 \%$ & 0.809 \\
\hline & No & 108 & 85 & $78.7 \%$ & 23 & $21.3 \%$ & \\
\hline \multirow[t]{3}{*}{ Barriers in changing life style } & Economic & 39 & 30 & $76.9 \%$ & 9 & $23.1 \%$ & 0.895 \\
\hline & Social & 27 & 22 & $81.5 \%$ & 5 & $18.5 \%$ & \\
\hline & No barriers & 162 & 129 & $79.6 \%$ & 33 & $20.4 \%$ & \\
\hline \multirow[t]{2}{*}{ Add sugar to tea/coffee? } & Yes & 82 & 64 & $78.0 \%$ & 18 & $22.0 \%$ & 0.708 \\
\hline & No & 146 & 117 & $80.1 \%$ & 29 & $19.9 \%$ & \\
\hline \multirow[t]{4}{*}{ Usual lunch } & Rice & 158 & 124 & $78.5 \%$ & 34 & $21.5 \%$ & 0.004 \\
\hline & Bread & 12 & 12 & $100.0 \%$ & 0 & $0.0 \%$ & \\
\hline & Other & 21 & 21 & $100.0 \%$ & 0 & $0.0 \%$ & \\
\hline & Don't take lunch & 37 & 24 & $64.9 \%$ & 13 & $35.1 \%$ & \\
\hline \multirow[t]{4}{*}{ Usual dinner } & Rice & 101 & 84 & $83.2 \%$ & 17 & $16.8 \%$ & 0.466 \\
\hline & Bread & 13 & 10 & $76.9 \%$ & 3 & $23.1 \%$ & \\
\hline & Other & 38 & 31 & $81.6 \%$ & 7 & $18.4 \%$ & \\
\hline & Don't take dinner & 76 & 56 & $73.7 \%$ & 20 & $26.3 \%$ & \\
\hline \multirow[t]{5}{*}{ Frequency of eating bread } & More than once a day & 62 & 45 & $72.6 \%$ & 17 & $27.4 \%$ & 0.122 \\
\hline & Once a day & 45 & 33 & $73.3 \%$ & 12 & $26.7 \%$ & \\
\hline & More than once a week & 31 & 25 & $80.6 \%$ & 6 & $19.4 \%$ & \\
\hline & Once a week & 68 & 61 & $89.7 \%$ & 7 & $10.3 \%$ & \\
\hline & Less than once a week & 22 & 17 & $77.3 \%$ & 5 & $22.7 \%$ & \\
\hline \multirow[t]{4}{*}{ Frequency of eating rice } & Never & 25 & 24 & $96.0 \%$ & 1 & $4.0 \%$ & 0.044 \\
\hline & Once a day & 35 & 31 & $88.6 \%$ & 4 & $11.4 \%$ & \\
\hline & Twice a day & 57 & 42 & $73.7 \%$ & 15 & $26.3 \%$ & \\
\hline & Three times a day & 111 & 84 & $75.7 \%$ & 27 & $24.3 \%$ & \\
\hline \multirow[t]{2}{*}{ Rice type } & Red & 112 & 100 & $89.3 \%$ & 12 & $10.7 \%$ & $<.001$ \\
\hline & White & 116 & 81 & $69.8 \%$ & 35 & $30.2 \%$ & \\
\hline \multirow[t]{2}{*}{ Physical activities during past month } & Yes & 209 & 174 & $83.3 \%$ & 35 & $16.7 \%$ & $<.001$ \\
\hline & No & 19 & 7 & $36.8 \%$ & 12 & $63.2 \%$ & \\
\hline \multirow[t]{4}{*}{ Frequency of eating fruit } & At least twice a day & 41 & 36 & $87.8 \%$ & 5 & $12.2 \%$ & 0.007 \\
\hline & At least once a day & 82 & 72 & $87.8 \%$ & 10 & $12.2 \%$ & \\
\hline & More than once a week & 54 & 36 & $66.7 \%$ & 18 & $33.3 \%$ & \\
\hline & Less than or equal to once a week & 51 & 37 & $72.5 \%$ & 14 & $27.5 \%$ & \\
\hline \multirow[t]{3}{*}{ Exercise time } & None & 135 & 96 & $71.1 \%$ & 39 & $28.9 \%$ & 0.040 \\
\hline & Less than 30 minutes & 21 & 20 & $95.2 \%$ & 1 & $4.8 \%$ & \\
\hline & Greater than or equal to 30 minutes & 23 & 19 & $82.6 \%$ & 4 & $17.4 \%$ & \\
\hline \multirow[t]{3}{*}{ Exercise in leisure time } & Not at all & 135 & 96 & $71.1 \%$ & 39 & $28.9 \%$ & 0.002 \\
\hline & Less than once a month & 12 & 10 & $83.3 \%$ & 2 & $16.7 \%$ & \\
\hline & Less than once a week & 38 & 35 & $92.1 \%$ & 3 & $7.9 \%$ & \\
\hline
\end{tabular}


Table 1 (continued)

\begin{tabular}{|c|c|c|c|c|c|c|c|}
\hline \multicolumn{3}{|l|}{ Variables } & \multicolumn{5}{|c|}{ EMA (Emergency Medical Assistance) } \\
\hline & & & \multicolumn{2}{|c|}{0 Admission } & \multicolumn{3}{|c|}{$>=1$ Admission } \\
\hline \multirow[b]{2}{*}{ Smoke } & & 43 & 40 & $93.0 \%$ & 3 & $7.0 \%$ & \\
\hline & Never & 204 & 163 & $79.9 \%$ & 41 & $20.1 \%$ & 0.263 \\
\hline \multirow{5}{*}{ Alcohol } & Given up temporarily or permanently & 10 & 9 & $90.0 \%$ & 1 & $10.0 \%$ & \\
\hline & Less than or equal to daily & 14 & 9 & $64.3 \%$ & 5 & $35.7 \%$ & \\
\hline & Never & 202 & 161 & $79.7 \%$ & 41 & $20.3 \%$ & 0.339 \\
\hline & Given up temporarily or permanently & 15 & 13 & $86.7 \%$ & 2 & $13.3 \%$ & \\
\hline & Less than or equal to daily & 11 & 7 & $63.6 \%$ & 4 & $36.4 \%$ & \\
\hline \multirow{2}{*}{\multicolumn{3}{|c|}{ Continuous variables }} & \multicolumn{2}{|c|}{0 Admission } & \multicolumn{2}{|c|}{$>=1$ Admission } & $p$ value \\
\hline & & & Mean & $\mathrm{SD}$ & Mean & $\mathrm{SD}$ & \\
\hline \multicolumn{3}{|l|}{ BMI } & 30.99 & 7.53 & 34.07 & 6.39 & 0.011 \\
\hline \multicolumn{3}{|c|}{ Waist Hip Ratio } & 0.78 & 0.17 & 0.82 & 0.13 & 0.051 \\
\hline \multicolumn{3}{|c|}{ Fasting Blood Glucose Level (mmol) } & 6.30 & 2.23 & 7.45 & 2.56 & 0.002 \\
\hline \multicolumn{3}{|l|}{ Age } & 62.12 & 9.30 & 64.30 & 10.95 & 0.169 \\
\hline \multicolumn{3}{|c|}{ Time duration since diagnosis of diabetes } & 9.92 & 4.07 & 9.68 & 3.08 & 0.711 \\
\hline \multicolumn{3}{|c|}{ How many meals do you usually eat? } & 3.04 & 0.32 & 3.00 & 0.47 & 0.508 \\
\hline
\end{tabular}

performed to test associations between DREMA and the 18 categorical variables. $t$ test was performed on the six continuous variables; (1) BMI, (2) waist-to-hip ratio (WHR), (3) FBG, (4) age, (5) time duration since diagnosis, and (6) number of meals per day. Finally, the DREMA prediction model was developed based on parameter estimates of a logistic regression model on DREMA ( $\geq$ 1 admission vs. 0 admissions), with variable selection from a forward stepwise selection process, considering all 24 potential independent variables. In the forward selection procedure, each variable was entered into the model one at a time, and the most significant variable was selected to enter into the model first. The remaining variables were then tested again one at a time, with inclusion of the next most significant variable, and so-on until all significant variables had been added $(p<0.05)$. Table 2 provides the logistic regression for the retained variables. Receiver operating characteristic (ROC) curve was calculated for each model. Area under the curve (AUC) used to compare the ability of the model to accurately predict DREMA; $\mathrm{AUC}=1$ indicates a perfect predictor and $\leq 0.5$ a worthless predictor [21]. Youden's Index, $J$ $(J=$ sensitivity + specificity -100$)$, identified optimum cutoff for the probability of DREMA seeking, which best classified patients as DREMA seeking and not seeking. Youden's Index is commonly used as a summary measure of the ROC curve, because it can identify the maximum potential effectiveness of a biomarker [22].

\section{Results}

Of the 228 respondents (mean age $=62.6$ years), $113(49.6 \%)$ were female and $115(50.4 \%)$ were male. Majority of the participants did not have high school diplomas $(74.1 \%)$ and were either retired $(53.1 \%)$ or unemployed $(24.1 \%)$. Pearson Chi-square tests $\left(\chi^{2}\right)$ for the 18 categorical variables showed that seven distinct variables were statistically significant $(p<0.05)$ in predicting DREMA: (1) type of lunch $\left(\chi^{2}(3)=13.41, p=0.004\right),(2)$ frequency of eating rice $\left(\chi^{2}(3)=8.09, p=0.044\right),(3)$ rice type $\left(\chi^{2}(3)=13.18\right.$, $p<0.001)$, (4) physical activities $\left(\chi^{2}(3)=22.93, p<0.001\right)$, (5) frequency of fruit intake $\left(\chi^{2}(3)=12.12, p=0.007\right)$, (6) duration of exercise time $\left(\chi^{2}(3)=6.44, p=0.040\right)$, and (7) frequency of exercise on leisure time $\left(\chi^{2}(3)=14.41\right.$, $p=0.002)$. Those who did not eat lunch were most likely to seek DREMA (35.1\%) followed by those who ate rice $(21.5 \%)$, compared with those eating bread or "Other" $(0 \%)$. Subjects who ate rice two or three times a day required DREMA more often (26.3 and $24.3 \%$, respectively) than those who never ate rice $(4 \%)$ or ate it once a day $(11.4 \%)$. Those eating white rice were more likely to seek DREMA $(30.2 \%)$ than those eating red rice $(10.7 \%)$. Respondents who reported not exercising regularly were more likely to seek DREMA (28.9\%) than those who exercised.

No significant association existed between DREMA and the remaining 11 variables; gender, posture at work, having regular clinic visits, diabetes education, barriers in changing lifestyle, adding sugar to tea/coffee, type of dinner, frequency of eating bread, educational level, smoking, and alcohol use.

DREMA demonstrated a significant positive association with FBG $(t(226)=3.06, p=0.002)$ and BMI $(t(226)=2.58, p=0.011)$, where higher FBG $(\mathrm{M}=7.45$, $\mathrm{SD}=2.56)$ and higher $\mathrm{BMI}(\mathrm{M}=34.1, \mathrm{SD}=6.39)$ were found 
Table 2 Logistic Regression on DREMA ( $>=1$ admission vs 0 admissions) (N=228)

\begin{tabular}{|c|c|c|c|c|c|c|}
\hline & Beta & S.E. & Wald & df & $p$-value & Odds Ratio \\
\hline Age & 0.07 & 0.02 & 9.07 & 1 & 0.003 & 1.07 \\
\hline Rice Type (White vs Red) & 1.22 & 0.43 & 8.20 & 1 & 0.004 & 3.39 \\
\hline Any Physical Activity outside regular job (No vs Yes) & 3.91 & 0.85 & 21.15 & 1 & $<.0010$ & 49.90 \\
\hline Leisure Exercise: Less than once a month (Level 2) vs Not at all (Level 1) & -0.29 & 0.86 & 0.11 & 1 & 0.736 & 0.75 \\
\hline Leisure Exercise: Less than once a week (Level 3) vs Not at all (Level 1) & -1.63 & 0.69 & 5.61 & 1 & 0.018 & 0.20 \\
\hline Leisure Exercise: Less than or equal to everyday (Level 4) vs Not at all (Level 1) & -3.55 & 1.12 & 10.00 & 1 & 0.002 & 0.03 \\
\hline
\end{tabular}

among respondents with at least one admission compared with those with no admissions (FBG: $\mathrm{M}=6.30, \mathrm{SD}=2.23$; $\mathrm{BMI}$ : $\mathrm{M}=30.99$, SD = 7.53).

However, WHR, time duration since diagnosis, and the number of meals per day were not significantly important in predicting DREMA.

For the final DREMA prediction model, four variables were retained through a forward stepwise logistic regression analysis: (1) age, (2) the type of rice, (3) physical activity outside of a regular job (yes/no), and (4) exercise in leisure time (not at all, once a month or less, once a week or less, every day). The likelihood of seeking DREMA increased with aging, where those who were 10 years older were twice as likely to seek DREMA $\left(\mathrm{OR}=\mathrm{e}^{\wedge}(0.07 \times 10)=2.01\right)$ than someone 10 years younger. Regarding the type of rice, the odds of seeking DREMA increased with regular or frequent consumption of white rice rather than brown or parboiled rice. Thus, a patient who regularly eat white rice tended to have 3.39 times the odds of seeking DREMA compared with those who regularly eat brown or parboiled rice. With respect to patients' physical activity levels, being physically inactive outside of occupation aggravated the unfavorable outcomes associated with increasing age and increased the likelihood of seeking DREMA. Specifically, patients who did not engage in any physical activities outside of occupational posture during the past month had 50 times higher odds of seeking DREMA compared with those who regularly participated in physical activities during the same time duration. Also, the odds of seeking DREMA were directly associated with the frequency of exercise during leisure time. That is, a patient with higher frequency of leisure time exercise had lesser odds of seeking DREMA. For example, compared with those who exercised almost every day during their leisure time for the past 1 month (leisure exercise level 4), patients who did not exercise at all during their leisure time (leisure exercise level 1) had a 33.33 times higher odds of seeking DREMA. The final DREMA prediction model selected can be presented as a Y-prediction equation in the form of $\left(y=a+b^{* \times 1}+c^{* \times 2}+d^{* \times 3}+e^{* \times 4}\right)$, where $y$ is on the logit scale to model the probability of DREMA, $a$ through $e$ are the parameter estimates from the logistic regression model, and $\times 1$ through $\times 4$ are each respondent's data values for the four independent variables in the model:

$$
\begin{aligned}
Y=\log \left(\frac{p}{1-p}\right)= & -2.53+0.07 \times(\text { age })+3.91 \\
& \times(\text { physically inactive })+1.22 \\
& \times(\text { white rice consumption })-0.291 \\
& \times(\text { leisure activity level } 2)-1.63 \\
& \times(\text { leisure activity level } 3)-3.55 \\
& \times(\text { leisure activity level } 4)
\end{aligned}
$$

To calculate the probability of DREMA, $p=\exp (Y) /(1+$ $\exp (Y))$.

The probability of a given patient seeking DREMA, i.e., $\mathrm{P}(\mathrm{DREMA})$, can be calculated using the equation $p=\exp (Y) /$ $(1+\exp (Y))$.

Finally, the AUC value for this DREMA-seeking prediction model was 0.812 (Fig. 1), indicating a good classification accuracy. Using Youden's Index, the optimal cutoff point for the probability of DREMA seeking based on the prediction model is 0.285 . Thus, a predicted probability of 0.285 or above was classified as seeking DREMA, with moderate sensitivity and high specificity values of 0.66 and 0.81 , respectively [23].

\section{Discussion}

Overall, the final DREMA prediction model was both meaningful and simple (user-friendly), and with integration of a tool (e.g., computer program) to help with calculations, may prove useful to both clinicians and non-clinicians. With further validation and updating of this model (and its underlying concept) based on characteristics of local populations, 


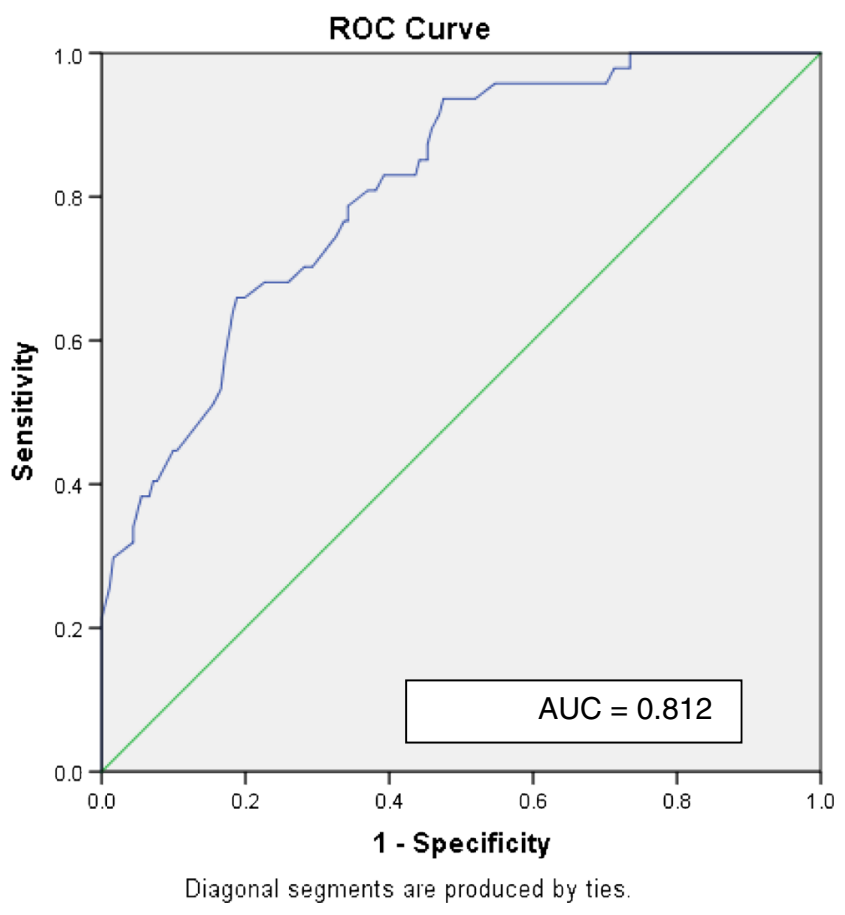

Fig. 1 AUC value for DREMA-seeking prediction model

healthcare providers will be able to predict the likelihood of seeking DREMA for each clinic patient diagnosed with diabetes.

Several difficulties were encountered during this study. Firstly, absence of a control group (e.g., non-daibetic patients) made it impossible to investigate DREMA seeking by persons who were not diagnosed with diabetes as well as those who were diagnosed with diabetes and its common cardiovascular complications. Secondly, data were missing for six variables (removed) either because a patient refused to report them or because the interviewer failed to collect the information from a patient with adequate clarity. Student $t$ test was performed to compare means for patients with complete data profiles and those with missing data, which excluded the existence of any systematic difference $[24,25]$. Thirdly, many variables that were not significant in bivariate analysis may have multidimensional constructs that are not easy to quantify. Put differently, the real-world scenario would be far more complicated than the developed conceptual model [24]. Fourthly, the study failed to collect data on patients who had not been discharged from the hospital, were terminally ill, or had died following a DREMA event during the study period because only those who visited the clinic were investigated. In order to validate the proposed model, further iterations of this study may use a prospective approach. Moreover, the model may not be sensitive to patients with either cardiovascular complications or other comorbidities (e.g., asthma, epilepsy) and those with type-1 diabetes since patients with such comorbidities were excluded from the study. Therefore, the safer way of using the prediction model would be recognizing low-risk patients rather than leaving the model to identify high-risk patients. Thus, future research should attempt to validate the model in a realworld scenario involving patients with cardiovascular complications and other diabetes-related comorbidities.

Finally, model selection was based on statistical significance and meaningfulness of the combination of variables. Generalizing to a different community may require model updating based on unique characteristics of the local population [25].

Alternative methods, such as artificial neural networks and decision trees, may also be useful to clinicians for predicting DREMA events. Artificial neural networks are computational models, which resemble neurons that generate an action potential based on an input from other neurons of the network [26]. Software of the artificial neural network interprets multiple inputs fed into it and produces a dichotomous output. Both input data and outcomes help the neural network to implicitly identify complex linear or non-linear relations and possible interactions between predictor and outcome variables in order to calculate the probability of a specific outcome [27-29]. However, artificial neural networks have several limitations, such as restricted ability to explicitly identify causal relationships, not being user-friendly, greater need for computational resources and training, and tendency to effectively memorize irrelevant data (overfitting) that reduces predictive accuracy [27]. A mathematical algorithm, which is set to maximize the predictive accuracy, produces variables, cutoff values, and suitable sequence of splitting, resulting in a "decision tree" that can be easily translated into routine clinical practice [14]. Clinicians can predict the outcome for patients by simply following the answers to questions in each of the boxes [30]. However, this method can be less accurate than other prediction models because of the limited number of variables available on the trees [31].

Several new questions arise from this study. The attitude of clinicians toward using the model is critical and must be verified. Finally, model selection was based on statistical significance and meaningfulness of the combination of variables. Research should investigate model applicability in different types of communities [25], and, if not applicable, determine required modifications based on unique characteristics of the local population, e.g., anthropometric indicators, educational attainment, diabetes education, food habits, and physical activity. An impact analysis is required to measure the usefulness of the prediction model in the clinical practice with regard to patient satisfaction, cost-benefit, and time/resource constraints [10].

Findings of this study may influence clinical practice; factors associated with increasing the likelihood of DREMA events in individual patients were identified, suggesting interventions that might prevent those undesired events. In 
particular, increasing the level of physical activity at home, implementing a weight-loss plan to reduce central obesity (WHR), executing a dietary plan to prevent hyper- and hypoglycemia, consuming brown or parboiled rice as a family habit, and performing physical exercises five times per week, either singly or in combination, are useful behavioral interventions that may reduce the probability of DREMA events for individual patients.

Acknowledgements We would like to thank Dr. Ahmed YoussefAgha and Jessie He for their contributions to the first draft of the manuscript and data analysis, respectively.

Funding disclosure The authors have no relevant financial or nonfinancial relationships to disclose i.e., there was no funding or support.

\section{Compliance with ethical standards}

Conflict of interest The authors declare that they have no conflict of interest.

Ethical approval Ethical approval for the recruitment of human subjects and review of medical records were obtained from the Institutional Review Board, Indiana University Bloomington, USA.

Informed consent The authors obtained informed consent from all individual participants included in the study.

\section{References}

1. Fox CS, Coady S, Sorlie PD, et al. Trends in cardiovascular complications of diabetes. JAMA-J Am Med Assoc. 2004;292(20): 2495-9.

2. Ritz E, Orth SR. Nephropathy in patients with type 2 diabetes mellitus. N Engl J Med. 1999;341(15):1127-33.

3. Sumner CJ, Sheth S, Griffin JW, Cornblath DR, Polydefkis M. The spectrum of neuropathy in diabetes and impaired glucose tolerance. Neurology. 2003;60(1):108-11.

4. Ramsey SD, Sandhu N, Newton K, et al. Incidence, outcomes, and cost of foot ulcers in patients with diabetes. Diabetes Care. 1999;22(3):382-7.

5. Nyenwe EA, Kitabchi AE. Evidence-based management of hyperglycemic emergencies in diabetes mellitus. Diabetes Res Clin Pract. 2011;94(3):340-51.

6. Nita CA, Rusu A, Bala CG, Hancu N. Predictors of postprandial hyperglycemia in patients with type 2 diabetes. Acta Endocrinol. 2009;5(2):177-82.

7. Di Cianni G, Goretti C, Onetto F, et al. Emergency hospitalizations for severe hypoglycaemia in patients with type 2 diabetes. Acta Diabetol. 2013;50(3):463-4.

8. Begum N, Donald M, Ozolins IZ, Dower J. Hospital admissions, emergency department utilisation and patient activation for selfmanagement among people with diabetes. Diabetes Res Clin Pract. 2011;93(2):260-7.

9. National Trauma Secretariat of Sri Lanka. Emergency medical services. 2013; http://www.traumaseclanka.gov.lk/. Accessed 12 November 2013.
10. Adams ST, Leveson SH. Clinical prediction rules. Br Med J 2012;344.

11. Liao L, Mark DB. Clinical prediction models: are we building better mousetraps? J Am Coll Cardiol. 2003;42(5):851-3.

12. Gandara E, Wells PS. Diagnosis: use of clinical probability algorithms. Clinics in Chest Medicine. 2010;31(4):629.

13. Marchese MC. Clinical versus actuarial prediction - a review of the literature. Percept Mot Skills. 1992;75(2):583-94.

14. Grobman WA, Stamilio DM. Methods of clinical prediction. Am J Obstet Gynecol. 2006;194(3):888-94.

15. Bland JM, Altman DG. Statistics notes - the odds ratio. Br Med J. 2000;320(7247):1468-8.

16. Reed M, Huang J, Brand R, et al. Implementation of an outpatient electronic health record and emergency department visits, hospitalizations, and office visits among patients with diabetes. JAMAJournal of the American Medical Association. 2013;310(10): 1060-5.

17. American Diabetes Association. Standards of medical care in diabetes-2011. Diabetes Care. 2011;34(Supplement 1):S11-61.

18. Amarasinghe DACL, Fonseka P, Dalpatadu KCS, Unwin NC, Fernando DJS. Risk factors for long-term complications in patients with type 2 diabetes attending government institutions in the Western Province of Sri Lanka: a case control study. Diabetes Res Clin Pract. 2006;75:377-8.

19. Shrivastava S, Shrivastava P, Ramasamy J. Role of self-care in management of diabetes mellitus. J Diabetes Metab Disord. 2013;12(1):14.

20. Morris SK, Parkin P, Science M, et al. A retrospective crosssectional study of risk factors and clinical spectrum of children admitted to hospital with pandemic H1N1 influenza as compared to influenza A. BMJ Open. 2012;2(2).

21. Zweig MH, Campbell G. Receiver-operating characteristic (ROC) plots - a fundamental evaluation tool in clinical medicine. Clin Chem. 1993;39(4):561-77.

22. Ruopp MD, Perkins NJ, Whitcomb BW, Schisterman EF. Youden index and optimal cut-point estimated from observations affected by a lower limit of detection. Biometrical J Biometrische Zeitschrift. 2008;50(3):419-30.

23. Šimundić A-M. Measures of diagnostic accuracy: basic definitions. EJIFCC. 2009;19(4):203-11.

24. Tabachnick BG, Fidell LS. Using multivariate statistics. 6th ed. Boston: Allyn and Bacon; 2013.

25. Schoenbach V, Rosamond W. Understanding the fundamentals of epidemiology: an evolving text. In: Department of Epidemiology SoPH UoNCaCH. Fall 2000 ed. Chapel Hill: University of North Carolina; 2000. p. 209-20.

26. Buscema M. A brief overview and introduction to artificial neural networks. Substance Use Misuse. 2002;37(8-10):1093-148.

27. Tu JV. Advantages and disadvantages of using artificial neural networks versus logistic regression for predicting medical outcomes. J Clin Epidemiol. 1996;49(11):1225-31.

28. Westreich D, Lessler J, Funk MJ. Propensity score estimation: neural networks, support vector machines, decision trees (CART), and meta-classifiers as alternatives to logistic regression. J Clin Epidemiol. 2010;63(8):826-33.

29. Hermundstad AM, Brown KS, Bassett DS, Carlson JM. Learning, memory, and the role of neural network architecture. Plos Computational Biology. 2011;7(6).

30. Tsai C-L, Clark S, Camargo CA Jr. Risk stratification for hospitalization in acute asthma: the CHOP classification tree. Am J Emerg Med. 2010;28(7):803-8.

31. Bellazzi R, Zupan B. Predictive data mining in clinical medicine: current issues and guidelines. Int J Med Inform. 2008;77(2):81-97. 